\title{
Evaluation of permanent deformation characteristics of unmodified and Polyethylene Terephthalate modified asphalt mixtures using dynamic creep test
}

Taher Baghaee Moghaddam, Mehrtash Soltani, Mohamed Rehan Karim

Introduction

Plastics have prominent utilization worldwide. Polyethylene Terephthalate (PET) is one of the major types of plastics that can be found in the Municipal Solid Waste (MSW) [1]. MSW contains high proportion of thermoplastic polymers which has been increasing in value. PET is a semi-crystalline thermoplastic polymer, and is considered as polyester material [2]. Large amount of waste PET is being produced in the form of different products, for instance, bottles, fibers, molding and sheets are the products mostly manufactured in Europe by application of PET [3]. Utilization of PET is wel1-known in food industry because of superlative characteristics offered by PET as a packaging material, mainly as bottles [4].

During the last decades, number of vehicles on roads, especially heavy vehicle such as vans and trucks, has been increased, considerably. By rising the number and weight of vehicles, amount and cycles of applied loads to the asphalt mixture has increased significantly, thus service life of asphalt mixture was decreased. Moreover, performance of asphalt mixture is influenced by ambient temperature due to asphalt' s visco-elastic properties. Asphalt viscosity is highly affected by temperature when higher temperature results in softer and less viscose asphalt binder which can affect the permanent deformation performance of asphaltic concrete in negative manner.

To overcome these problems and make a better mixture providing longer service life, modifying asphalt mixture by utilizing additives such as fibers and polymers comes into popularity among road engineers and designers [5]. Among these additives, polymers 
(such as SBS, LDPE, and HDPE) have a prominent utilization [6-8].

In this case, many investigations have been conducted on effectiveness

of using waste materials as additives in order to: (1) prevent

from imposed additional charges by using additives and, (2) find a

solution to reuse waste materials as secondary materials in such

construction projects [9-12].

Hence, this study aims to assess effects of adding waste PET as

an additive on permanent deformation characteristics of asphalt

mixture.

\section{Background}

Progressive accumulation of permanent deformation is defined

as rutting. Rutting, which is caused by repeated traffic loading, is

sum of the total deformation accrues in each layer of pavement structure and, moreover, asphalt layer has shown a

prominent magnitude in rutting [13]. The permanent deformation of asphalt

mixture is greatly influenced by type of asphalt mixture and percentage

of voids in mix [5]. In this case, it was reported that stone

mastic asphalt (SMA) mixture has better performance in comparison

with conventional dense graded mixture $[14,15]$.

Different test methods can be used to evaluate permanent

deformation of asphalt mixture such as static and dynamic creep

tests as well as wheel tracking test among which static test was

estimated that cannot be a true indicator of permanent deformation

of modified mixture; however, dynamic creep test is considered

as one of the best test procedure to assess the permanent

deformation of asphalt mixture $[16,17]$. Concept of this method

which is based on axial compression was developed in 1970 by

Monismith et a1. [18]. Further, it is reported that among all test procedures

dynamic creep test is an appropriate laboratory method to

investigate permanent deformation performance of modified and

unmodified asphalt layers [19].

Several permanent deformation models were proposed since

1970s that include power-1aw mode1, VESYS mode1, Ohio State

mode1, superpave and AASHT0 2002 mode1s [18, 20,21]. In past researches,

Flow Number (FN) was a popular indicator to assess the

permanent deformation of hot mix asphalt [22,23]. This value can

be obtained as result of dynamic test by plotting cumulative plastic

strain vs number of load cycles. 
There are three phases of flow during the test namely: primary, secondary and tertiary stages. During the primary stage rate of strain decreases by load application. In secondary phase, rate of strain almost remains constant, and during tertiary phase the strain rate rises dramatically which is due to significant deformation of specimen under cyclic load application. In this criterion, the FN is defined as the load cycles when the tertiary stage is initiated. Although this method is one of the criteria to assess rutting performance of asphalt mixture, it has a disadvantage because it is observed that several minimum values from the strain rate were found $[20]$.

In later investigation, in 2004, another model was developed by Zhou et al. [22]. Zhou proposed three-stage model with one model for each stage of creep curve namely: power-law model, linear model and exponential model each for primary, secondary and tertiary stages, respectively (Fig. 1). The models are presented at follows:

Full text available at :

http://www.sciencedirect.com/science/article/pii/S0261306913006390 